\title{
A New Method for Measuring the Angle of Hallux Valgus
}

\author{
${ }^{1}$ Rahmat allah Heidarpour ${ }^{*}$, ${ }^{1}$ Morteza Abdiani \\ ${ }^{1}$ Department of Sport and Exercise Science, Faculty of Humanities, University of Arak, Arak, Iran.
}

\begin{abstract}
Objectives. The aim of this study is to introduce a new way and assign its reliability and validity in measuring the angle of hallux valgus.

Methods. In this method, we have proceeded to introduce a simple way for measuring the angle of hallux valgus by using ruler and mathematical formulas. For doing this study, we selected 25 students and measured their angle of hallux valgus with new way and by goniometer. To assign the reliability and validity, it has been used of correlation coefficient and ICC coefficient, respectively.

Results. Findings show that, there is a significant correlation between new method and using goniometer ( $\mathrm{r}=0 / 825)$. Besides, this method is of the high reliability for measuring the angle of hallux valgus (ICC=0/992).

Conclusion. The new method not only is both cheap and simple measuring for measuring the angle of hallux valgus, but also have high reliability and validity.
\end{abstract}

KEY WORDS: Hallux Valgus, Goniometer, Angle of Hallux, Law of Cosines.

\section{INTRODUCTION}

Hallux valgus is a kind of deformity on the hallux and first metatarsal. Aetiology of this deformity could be explained as either heritage or environment factors $(1,2)$, such as gene inheriting or wearing the unfit footwear or even the problem of obesity. Prior to the correct diagnosis of hallux valgus, it is important to define a way to measure it (3). One of the most important indicators of development of a community is the health and well-being of its individuals. Health of any society depends on the physical and mental health of its individuals, particularly adolescents and young people. Naturally, having healthier and more dynamic people would result in a more capable society. In the new era, although technology has brought welfare for human, its unfavorable consequences are inevitable. One of the most important problems is the replacement of muscular force with machines, resulting immobility, inactivity, obesity, abnormalities and diseases. Immobility leads to negative consequences in the long-term. The results of research conducted in Iran showed a high prevalence of disorders among the population, so that researchers consider it worrisome $(4,5)$. During e walk, toe thumb is the last axis that carries force to move the body. Therefore, any deviation in its natural state can lower the performance of walking. Normally, the hallux (big toe) has a seven degrees deviation with first metatarsal bone axis, called physiologic toe angle (6). If the deviation angle of the big toe becomes more than 10 degrees, it is considered as 'hallux valgus' deformity, commonly called as bunion. In more advanced stages, these disorders have also been

*. Corresponding Author:

Rahmat allah Heidarpour

E-mail: heidar_msi@yahoo.com 
reported higher than 30 degrees so that the anomaly puts pressure on the second finger $(6,7)$. Wearing narrow-toed shoes and high heels, flat foot, the loss of the transverse arch of the foot, weakness of adductor muscles or failure of anterior muscles of the thumb, hereditary factors, valgus deformity and arthritis are among the causes of bunion (7). With regard to the important role of the thumb in transferring weight during walking, little research is done in this context in Iran. In this regard, the researches show that this anomaly is one of the most common disorders among the students. Heidarpour et al. reported that the prevalence of this malformations is 47.3 percent among boy and girl students of Kohgiluyeh and Boyer Ahmad province, which was the highest percentage among the 10 anomalies that were measured (8). Fathi states the prevalence of these disorders in male and female students $19.38 \%$ (9).

Bunion diagnosis is often done by visual observation; however, X-ray is used for treatment and surgery. The vertical line is another method for diagnosing bunion. Vertical line passes normally through the first and second fingers, while in hallux valgus, vertical line passes through the thumb. Another tool for measuring bunion is a goniometer that measures the angle between the metatarsal bones and the first toe bone in degrees (10). It seems that visual and vertical line methods are not accurate methods for measuring the bunion. Using X-ray has restrictions such as high costs and complexity of the technique and the subject being exposed to X-rays. Among the above tools, goniometer is a suitable method for measuring bunion, but unavailability is the disadvantage of the method. Therefore, a new and available method is required so that people and especially teachers of physical education in schoolscould easily measure these anomalies and finally take steps to correct it. The aim of this study was to obtain a new method for measuring the bunion as well as the validity and reliability of it.

\section{MATERIALS AND METHODS}

Participants. This is an argumentative study that eventually is tested by means of comparative methods. 25 students of Kohgiluyeh and Boyer-
Ahmad province were purposefully selected; and their hallux valgus angle were measured by the goniometer.

New Method. In addition, in order to measure the angle of hallux valgus by the new method, measuring tape and mathematics formulas were used.

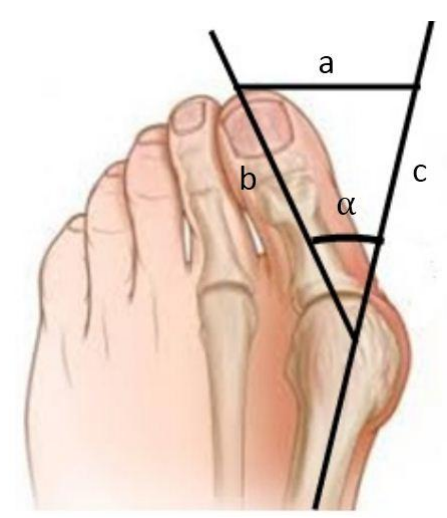

Figure 1. hallux valgus angle

The above figures shows the hallux valgus angle, on which a triangle is drawn. The angle between the big toe and the first metatarsal bone is also evident. In the new method we are trying to find the angle that deviates the big toe from the first metatarsal bone, using mathematical formulas. Below the above triangle is depicted. In this triangle, we can easily find any of the specified angles using the law of cosines. To get the angles of any triangle it is sufficient to measure its sides by a ruler or a measuring tape. Consider the following triangle,

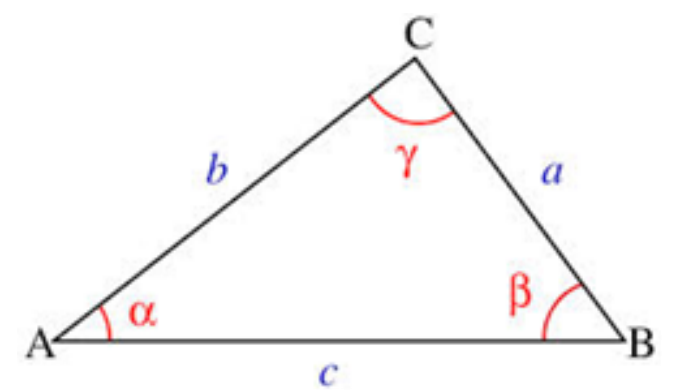

Law of cosines that is a useful mathematical theorem states that:

$$
a^{2}=b^{2}+c^{2}-2 b c(\cos \alpha) \text { (Equation 1) }
$$

Where $b$ and $c$ are the sides of the triangle, and $\alpha$ is the angle between two sides of $\mathrm{b}$ and $\mathrm{c}(11-12)$. 
The Equation (1) can also be written as follows.

$$
\cos \alpha=\frac{\left(a^{2}-b^{2}-c^{2}\right)}{2 b c} \quad \text { Equation (2) }
$$

If you carefully observe the triangle drawn on the big toe, it can be claimed that the two sides of $b$ and $c$ are equal because the length of big toe bone is always the same in all rotations. Therefore, in the Equation (2), we have $b=c$. As a result, Equation (2) can be written as Equation (3).

$$
\cos \alpha=\frac{\left(a^{2}-2 b^{2}\right)}{2 b^{2}}
$$

Equation (3)

And eventually, the angle $\alpha$ that is the angle of hallux valgus can be obtained using the Equation (4).

$$
\alpha=\arccos \left(\frac{\left(a^{2}-2 b^{2}\right)}{2 b^{2}}\right) \quad \text { Equation (4). }
$$

Now, if in the above triangle we measure the sides of a triangle with a ruler or a measuring tape, the Hallux VALGUS angle can also be easily found by using the law of cosines. In the above method, triangle's sides is the only factor of measurement error, that due to the use of a ruler or measuring tape, this error is very small. Therefore, using the mentioned arguments, the above methods can be used to measure the angle of Hallux Valgus. The researchers believe that this argument alone is enough to recommend this method for measuring the angle of hallux valgus, however, here we also check the validity and reliability of the new method.
Statistical Analysis. To obtain the validity of the new method, the Pearson correlation coefficient is used to measure the correlation between the measurements of the hallux valgus angle between the new method and the goniometer method. In addition, Intraclass correlation coefficient (ICC) is used to check the reliability. Measurements using the new method were performed three times, with 15minute interval time by two evaluators (to determine ICC), and the mean of the three measurements was considered as the angle of hallux valgus to be used for finding correlation with goniometer angle. In addition, the analysis of variance was used for comparing the average of three measurements by two evaluators. Statistical analysis was done using SPSS Version 15.

\section{RESULTS}

Table (1) shows the validity of the measurements using the new measurement method and goniometer. In this table, the average of three measurements was considered as the angle of hallux valgus in the new method. As it can be seen, there is a significant and high correlation between the new approach and the goniometer.

Table 2 show the ICC reliability in the measurements of the two evaluators. The reliability of the new method is between the maximum of 0.995 and the minimum of 0.987 with the average of 0.992 , which has a high reliability. In addition, the analysis of variance shows that there is no significant difference between the mean of the three measurements $(\mathrm{F}=0.461$ and $\mathrm{sig}=0.461)$.

Table 1. Pearson's correlation to check the validity of the new method

\begin{tabular}{ccccc}
\hline Variable & Average \pm SD & The correlation coefficient $(\mathrm{r})$ & $\mathrm{p}$ \\
\hline Angle in the new method & $18.242 \pm 3.788$ & & \multirow{2}{*}{0.825} & $0.0001^{*}$ \\
\cline { 1 - 2 } Angle with goniometer & $17.240 \pm 3.519$ & & & \\
\cline { 1 - 2 } *. Significant $(\mathrm{p}<0.05)$ & & &
\end{tabular}

Table 2. ICC reliability to determine the correlation between the evaluators

\begin{tabular}{cccc}
\hline \multicolumn{2}{c}{ The first evaluator $($ Mean \pm SD) } & The second evaluator ( Mean \pm SD) & \multirow{2}{*}{ ICC Factor } \\
\cline { 1 - 3 } First stage & Second stage & third stage & \\
\hline $18.214 \pm 3.757$ & $18.205 \pm 3.886$ & $18.307 \pm 3.812$ & 0.992 \\
\hline
\end{tabular}




\section{DISCUSSION AND CONCLUSION}

The results show that there is an acceptable high validity between the new method of hallux valgus angle measurement and the goniometer method. Although the goniometer is a good way to measure angles, but unavailability and its relatively high price is a disadvantage of the goniometer method. Table 2 also shows that the new method has an acceptable reliability. For the reliability of this method we can say that using a ruler or a measuring tape is a very reasonable and highly reliable method to measure the length of objects. In this method we used a ruler to measure the length of the big toe and distance of toe tip to where it deviates from it, which had a very low margin of error. In the next step we transferred the data to the SPSS software, and using this software and the mathematical formulas has no error in them.

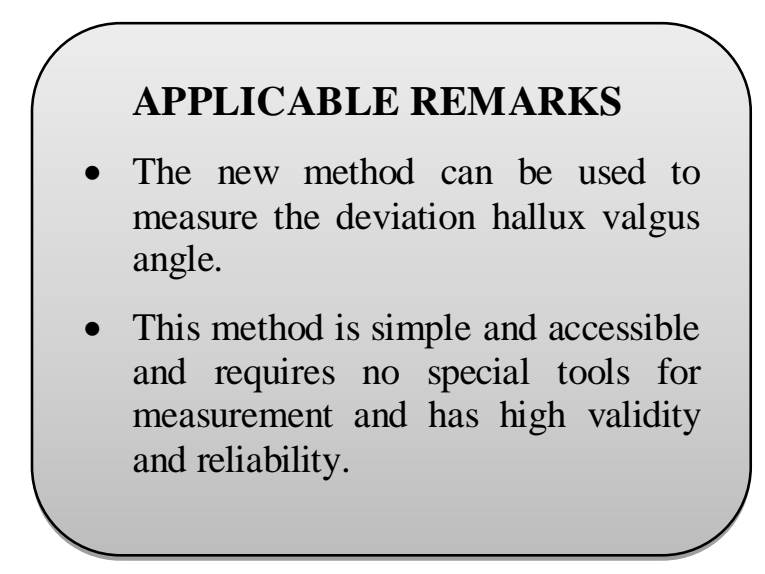

\section{REFERENCES}

1. Bonney G, Macnab I. Hallux valgus and hallux rigidus: a critical survey of operative results, J Bone Joint Surg Br, Vol.34, No. B, 1952, pp.366-85.

2. Brodie BS RC, Robins DJ, Wilson AFJ, Wessex Feet. a regional foot health survey, Volume I. The survey, The Chiropodist, Vol.43, 1988,pp.56-152.

3. Zhou J, Hlavacek P, Xu B, Chen W. Approach for measuring the angle of hallux valgus. Indian J Orthop 2013;47:278-82.

4. Heydari nik, Hossein. "Evaluation of physical abnormalities in secondary school students in Komijan city", Council for Educational Research. (2007).

5. Sene, Afsaneh. "Comparison of the prevalence of upper limb postural abnormalities in male and female secondary school students', Educational Innovation Quarterly, No. 30, Vol. VIII, 2009, pp. 139-156.

6. Letafat Kar K, Bakhshshi Haris M, Ghorbani S. "Corrective and remedial actions" Bamdad Ketab Publications. Second Edition. (2010). p(154-155).

7. Daneshmandi, H, Alizadeh, M H, Gharakhanlou R. "Corrective Movements". SAMAT Publications. Third Edition. (2005). p(136-138).

8. Heidarpour, Rahmatollah."Evaluating the Prevalence of stature abnormalities in male and female first and second grade High school students in Kohgiluyeh and BoyerAhmad Province. "Research project. YASD of Kohgiluyeh and BoyerAhmad Province. (2014).

9. Fathi, M. Rezai R. "Evaluation and Comparison of postural abnormalities in male and female middle school and high school students." Institute for Humanities and Cultural Studies. Eleventh Season, No. 1, 2010, pp. 46-53.

10. Heidari, F."Muscle weakness and correction movements and 1000 exercises" Shomal Payedar publication, Shomal Marashi Sports Complex, University of Shomal. $1^{\text {st }}$ edition. (2009). p(213-215).

11. Aitken, Mike, Bill Broadhurst, and Stephen Hladky. Mathematics for biological scientists. Garland Science, 2009 (page 106).

12. Waltham, David. Mathematics: a simple tool for geologists. John Wiley \& Sons, 2009. (page 75) 